PALABRAS CLAVE

Salud pública

Atención médica

Seguro de enfermedad

Financiación

Economía de la salud

Evaluación

Modelos matemáticos

Indicadores de salud

Brasil

Antônio M. Bós

Profesor Asociado, Tusculum College,

Greeneville, Tennessee

Investigador Adjunto,

Johns Hopkins Bloomberg School of

Public Health,

Departamento de salud internacional,

Baltimore, Maryland

œ abos@jhsph.edu

Hugh R. Waters

Profesor Asociado,

Johns Hopkins Bloomberg School

of Public Health,

Departamento de políticas

y gestión de salud,

Baltimore, Maryland

○waters@jhsph.edu
REVISTA DE LA CEPAL 95 A A OSTO 2008

\section{Efectos de protección financiera del sistema de salud pública y del seguro privado en Brasil}

\author{
Antônio M. Bós y Hugh R. Waters
}

$\mathrm{E}$

n este trabajo se evalúa la eficacia del sistema de salud pública y del seguro privado de salud en Brasil para dar protección financiera al cuidado de la salud. Los factores determinantes de los gastos catastróficos en salud se estiman mediante regresiones probit con control de ajustes de selección de Heckman en materia de necesidad de atención de salud. Se concluye que con el sistema público se reduce considerablemente (47\%) la probabilidad de que una familia tenga gastos de salud catastróficos y que con un seguro privado esto resulta $36 \%$ más probable. Las recomendaciones que se formulan apuntan al mejoramiento de la cantidad, accesibilidad, calidad y confiabilidad de los prestadores públicos de servicios de salud, a una provisión más adecuada de medicamentos por el sistema público y a una regulación más estricta del seguro privado de salud. 


\section{I}

\section{Introducción}

El concepto de protección financiera en materia de salud hace referencia al riesgo que representan los gastos de salud elevados para la estabilidad financiera de sectores vulnerables de la población. Las familias se pueden empobrecer si esos gastos sobrepasan su capacidad de pago (Gertler y Gruber, 2002; Strauss y Thomas, 1998; OMs, 2000). En los hogares más pobres hay menos capacidad para hacer frente a los problemas de salud, porque tienen menos activos líquidos para amortiguar la situación (Hulme y Shepherd, 2003). Un desafío crucial para el desarrollo es mejorar la capacidad de las familias pobres de resistir a estos embates, ya que de esa manera se acrecienta su potencial de obtener ingresos $\mathrm{y}$, por ende, se fomenta la inversión en capital humano y en actividades de mayor riesgo y más rentables (Banco Mundial, 2001).

Un método para evaluar la capacidad de protección financiera de un sistema de salud es determinar qué proporción de los domicilios tiene un gasto en salud que es elevado en comparación con su capacidad de pago (OMs, 2000; Waters, Anderson y Mays, 2004). En Kawabata, Xu y Carrin (2002) se propone la expresión "gastos de salud catastróficos" cuando los gastos de salud superan el $40 \%$ de la capacidad de pago de una familia, aunque se pueden usar umbrales diferentes. Según Xu, Evans y otros (2003), Brasil es el país de América Latina con la mayor proporción de hogares con gastos catastróficos $(10,3 \%)$. En una evaluación directa del efecto de los pagos relacionados con la salud sobre la pobreza, Murray, Xu y otros (2003) midieron la diferencia en el porcentaje de familias que se hallaban por debajo de la línea de pobreza antes y después de esos pagos. En Brasil esa diferencia era de 5,1 puntos porcentuales, y se encontraba entre las más elevadas de los 59 países estudiados.
La alta carga financiera asociada a la atención de la salud en Brasil está reñida con la concepción —o por lo menos la premisa- del Sistema Único de Salud (sus), que en principio es de acceso universal y gratuito en el lugar de la prestación. La ausencia de un estipendio u otros cargos pagaderos por el usuario debería reducir, o eliminar, la carga financiera inherente al cuidado de la salud, con la excepción de los impuestos necesarios para cubrir los costos del sistema. Sin embargo, el gasto de salud privado supera al gasto público (OPs, 2003) y el $25 \%$ de la población paga primas para contratar un seguro de salud (IBGE, 2000). Estas personas y familias efectivamente duplican su aporte, pues contribuyen indirectamente a los ingresos tributarios que financian el sus y directamente a los planes de seguros de salud complementarios. A diferencia de otros países, como Chile, las personas no tienen la opción de ser exoneradas del pago del financiamiento público si demuestran que tienen cobertura privada.

La aparente contradicción entre la alta carga financiera de la atención de salud en Brasil, por un lado, y los rasgos básicos del sus por el otro, resalta la importancia de estudiar los factores que determinan los gastos de salud catastróficos en el país. La capacidad del sistema público de reducir la carga financiera del cuidado de la salud es un importante indicador del desempeño del sistema (OMS, 2000). En el presente estudio se intenta explicar esa aparente contradicción y formular recomendaciones sobre cómo podría reformarse el sistema para mejorar su capacidad de protección financiera. Nuestro principal objetivo es determinar en qué forma el sus, el seguro privado de salud y el ingreso familiar influyen en la probabilidad de que una familia enfrente niveles catastróficos de gasto en salud. 


\section{II}

\section{La situación en Brasil: sistema de salud público y seguro de salud privado}

Desde que la Constitución Federal lo aprobó en 1998, Brasil tiene un sistema único de salud (sUs), con cobertura universal y gratuito para todas las personas en el lugar de atención. A diferencia de lo que ocurre en la mayoría de los países latinoamericanos, el sus no limita el acceso a quienes participan en el sistema de seguridad social. En este caso, las familias no tienen que decidir si adhieren o no al sus antes de afrontar una necesidad de atención de salud. También existe un sistema complementario privado que presta servicios de salud y que está integrado por grupos de organizaciones médicas, cooperativas médicas, seguros de salud y organizaciones de beneficencia. Los servicios privados pueden ser pagados directamente por los pacientes. Los beneficiarios de planes privados mantienen su pleno derecho a la cobertura del sus. En estas circunstancias, cuando una persona necesita una prestación de salud, decidirá si desea obtenerla a través del sus, a través de sus planes de seguro de salud privados o pagando directamente la atención privada (Bós y Bós, 2004).

El 76\% de la población es atendido exclusivamente por el sistema público de salud (ops, 2005, p. 13). Conforme a la legislación vigente, los tres niveles del gobierno - federal, estadual y local— deben participar en la financiación y el funcionamiento del sus, organizado en una red vinculada, regionalizada, jerárquica y descentralizada (OPS, 2007, p. 150). Las municipalidades tienen la responsabilidad primaria de brindar atención de salud a su población, con asistencia técnica y financiera del gobierno federal y de los estados.

El término "seguro privado" comprende los seguros que operan en el mercado y otros contratos de prepago obtenidos a través del empleo en el gobierno o en instituciones de propiedad del Estado, los seguros obtenidos a través del empleo privado y los seguros contratados directamente a la compañía aseguradora. Esta definición es similar a la usada por Sekhri y Savedoff (2006). Alrededor del $80 \%$ de los usuarios de la atención de salud complementaria recurre a grupos médicos, cooperativas médicas y seguros de salud (Fernández, Pires y otros, 2007). Entre quienes tienen seguro privado, el $57 \%$ lo obtuvo en su lugar de trabajo y los empleadores pagan parte de la prima, mientras que el $43 \%$ lo adquirió directamente de la aseguradora. En el 21\% de los planes de salud los pagos son conjuntos (IBGE, 2000). De la población cubierta por el sistema complementario, el $75 \%$ tiene cobertura de planes y seguros privados, mientras que el $25 \%$ está cubierto por sistemas que atienden a empleados públicos.

En 1998, el gasto total en salud nacional (público y privado) se estimaba en 7,9\% del producto interno bruto (PIB). El gasto público constituía el $41,2 \%$ de ese gasto total, es decir, 3,25\% del PIB. El nivel federal aportaba el $53 \%$ de los recursos, los estados el $22 \%$ y los municipios el $25 \%$. En la primera mitad de la década de 1990, la ausencia de fuentes estables de financiamiento federal llevó a una considerable pérdida de calidad de los servicios prestados por las instituciones públicas y los contratistas privados, la que solo comenzó a revertirse en 1995-1996. Los recursos obligatorios dispuestos por la enmienda de la Constitución $\mathrm{N}^{\circ} 29$ del año 2000 permitieron regularizar de manera razonable la afluencia financiera al sector de la salud, con importantes efectos en la capacidad de planificación a mediano y largo plazo (ops, 2005, p. 40).

El cuadro 1 muestra la extraordinaria expansión de la red de atención de salud pública y privada entre 1980 y 1999, particularmente para pacientes ambulatorios. La cantidad de servicios de salud sin hospitalización - unidades ambulatorias - se triplicaron con creces durante el período. Este fue un claro indicio de que comenzaba un cambio radical del modelo predominante hasta entonces, que estaba centrado en los hospitales (Escorel, Giovanella y otros, 2007; Gershman y Santos, 2006). Hasta mediados del decenio de 1980, los servicios curativos prestados por el Estado se concentraban en las ciudades y sus hospitales y estaban abiertos solamente a la minoría de trabajadores que laboraba en el sector formal. Como muestra el cuadro, en 1980 el $33 \%$ de los servicios de salud eran hospitales, cifra que en 1999 era solo de $16 \%$. Asimismo, si bien la mayoría de las unidades ambulatorias (73\%) son públicas, la mayor parte de los hospitales $(66 \%)$ son privados. Pero cerca del $83 \%$ de las camas de hospitales privados estaban a disposición del sus por contrato (OPS, 2005, p. 14).

El advenimiento del sistema de atención de salud universal redujo considerablemente la desigualdad de 
Brasil: servicios de salud, por tipo de atención y tipo de institución prestadora, 1980-1999

\begin{tabular}{|c|c|c|c|c|c|c|c|c|c|}
\hline \multirow{2}{*}{ Años } & \multicolumn{4}{|c|}{$\begin{array}{l}\text { Servicios de salud } \\
\text { con hospitalización }\end{array}$} & \multicolumn{4}{|c|}{$\begin{array}{l}\text { Servicios de salud } \\
\text { sin hospitalización }\end{array}$} & \multirow[t]{2}{*}{$\begin{array}{c}\text { Sin hospitalización } \\
(\%)\end{array}$} \\
\hline & Total & Público & Privado & Público (\%) & Total & Público & Privado & Público (\%) & \\
\hline 1980 & 6110 & 1217 & 4893 & 19,9 & 12379 & 8828 & 3551 & 71,3 & 67,0 \\
\hline 1999 & 7806 & 2613 & 5193 & 33,5 & 41009 & 29993 & 11016 & 73,1 & 84,0 \\
\hline Aumento (\%) & 16 & 114 & 6 & & 231 & 240 & 210 & & \\
\hline
\end{tabular}

Fuente: ops (2005).

acceso de los distintos grupos de población y de las diversas regiones del país. La proporción de hogares pobres con acceso a servicios de salud aumentó casi $50 \%$ entre 1992 y 2004 (Cornwall y Shankland, 2008). Como se ve en el cuadro 2, la distribución regional de camas de hospital muestra un perfil similar a la distribución de la población. Ha habido un crecimiento apreciable en la cantidad de consultas y hospitalizaciones por habitante en las regiones más pobres del país (la Norte y la Noreste). La puesta en práctica del sus condujo asimismo a una mejor distribución de los profesionales de la salud en el país, como muestra el cuadro 3. La proporción de médicos por cada 1.000 habitantes aumentó $54 \%$ a nivel nacional entre 1997 y el 2001. Aunque este crecimiento se observó en todas las regiones, persiste sin embargo una importante desigualdad: las regiones Sudeste, Sur y Centro-oeste tenían mucho más profesionales que la Noreste y la Norte.

Una importante iniciativa para ampliar la cobertura de la atención primaria de salud es el Programa de Salud Familiar (Escorel, Giovanella y otros, 2007), que brinda atención de salud integral en un territorio en particular, asignando pacientes a un equipo multidisciplinario compuesto por lo menos por un/a médico/a, un/a enfermero/a, auxiliares de enfermería y agentes comunitarios de salud. Cada equipo de salud es responsable de unas 1.000 familias. En el 2005, el programa se aplicaba en 4.837 municipalidades brasileñas, con 22.683 equipos de salud familiar que atendían a aproximadamente 73 millones de personas, es decir, el 40\% de la población (OPS, 2007, p. 147). Solla, Reis y otros (2007) señalan que el programa ha sido útil para reducir las desigualdades regionales en el acceso a la atención primaria.

Aun así, sigue habiendo profundas diferencias entre regiones en la prestación de servicios y el acceso a atención de salud intermedia y de alta complejidad. En el 2002, el $83 \%$ de los equipos ópticos, el $73 \%$ de
CUADRO 2

Brasil: distribución regional de camas de hospital y población, por regiones, 1999

\begin{tabular}{lcc}
\hline Regiones & $\begin{array}{c}\text { Porcentaje } \\
\text { de camas }\end{array}$ & $\begin{array}{c}\text { Porcentaje } \\
\text { de población }\end{array}$ \\
\hline Norte & 5,6 & 7,4 \\
Noreste & 26,1 & 28,2 \\
Sudeste & 43,6 & 42,6 \\
Sur & 16,7 & 14,9 \\
Centro-oeste & 8,0 & 6,8 \\
\hline
\end{tabular}

Fuente: ops (2005).

CUADRO 3

Brasil: número de profesionales de la salud, por regiones, 1997 y 2001

(Por cada mil habitantes)

\begin{tabular}{llllll}
\hline \multirow{2}{*}{ Regiones } & \multicolumn{2}{c}{ Médicos/as } & & \multicolumn{2}{c}{ Enfermeros/as } \\
\cline { 2 - 3 } \cline { 5 - 6 } & 1997 & 2001 & & 1997 & 2001 \\
\hline Brasil & 1,35 & 2,08 & & 0,45 & 0,52 \\
Norte & 0,61 & 1,12 & & 0,30 & 0,35 \\
Noreste & 0,80 & 1,20 & & 0,35 & 0,42 \\
Sudeste & 1,86 & 2,81 & & 0,54 & 0,60 \\
Sur & 1,36 & 1,99 & & 0,48 & 0,59 \\
Centro-oeste & 1,22 & 2,34 & & 0,37 & 0,47 \\
\hline
\end{tabular}

Fuente: OPS (2005).

los equipos de radioterapia, el 74\% de los equipos de resonancia magnética y el $75 \%$ de los equipos de tomografía computarizada del país estaban ubicados en las regiones Sur y Sudeste del país (OPS, 2005, pp. 20-21). En estas dos regiones reside el $57,4 \%$ de la población brasileña.

Pese a la persistente disparidad regional, ha habido una considerable mejora general en los indicadores de salud. El cuadro 4 muestra la evolución favorable del índice de mortalidad infantil y de esperanza de vida al 
CUADRO 4

Brasil: índice de mortalidad infantil y esperanza de vida al nacer, 1991-2006

\begin{tabular}{|c|c|c|c|}
\hline & 1991 & 2000 & 2006 \\
\hline $\begin{array}{l}\text { Índice de mortalidad infantil } \\
\text { (por mil) }\end{array}$ & 45,2 & 30,4 & 24,9 \\
\hline Expectativa de vida al nacer & 66,9 años & 70,4 años & 73,4 años \\
\hline
\end{tabular}

Fuente: IBGE ( 2006).

nacer. De Abreu, César y França (2007) encontraron que la mortalidad por causas evitables había disminuido desde que se echó a andar el sus y concluyeron que la disminución se había debido en parte a la mayor disponibilidad de servicios de salud y al mayor acceso a ellos que generó la reorganización del sistema de salud brasileño.

No hay cargos financieros asociados al uso del sistema público. Sin embargo, porque el sistema público excluye ciertos aspectos de la atención de salud (Ocké-Reis, Silveira y Andreazzi, 2002) o si la calidad (Costa y García, 2003), la accesibilidad y el tiempo de espera (Marinhol y Cardoso, 2007) de los prestadores públicos son inadecuados, es posible que las familias prefieran gastar más dinero en prestadores privados y seguro de salud privado (Ribeiro, Barata y otros, 2006; Bós, 2007). La expansión de los planes de salud privados en Brasil durante las décadas de 1980 y 1990 estuvo relacionada por lo menos parcialmente con la mala calidad de los servicios de los proveedores públicos (Alves y Timmins, 2003), la que se deterioró durante la década de 1980 luego de una serie de crisis económicas, crecimiento económico lento y escasez de recursos públicos (Malta, Cecílio y otros, 2004).
Casi todos los planes de salud privados incluyen las visitas médicas, los exámenes complementarios y las hospitalizaciones (oPs, 2005, p. 33). Según Fernández, Pires y otros (2007) los operadores de salud complementarios se concentran en las franjas de bajo valor de los planes de salud y los participantes buscan planes que presten servicios básicos. Al igual que en otros países con sistemas de salud universales, los planes privados de Brasil tienden a excluir el tratamiento de enfermedades crónicas que requieren cuidados prolongados y costosos y tienen estrictos límites en la extensión máxima de la hospitalización o el gasto total (Farias y Melamed, 2003; Pinto y Soranz, 2004; Rocha y Simões, 1999). Desde la perspectiva de un hogar asegurado, estas limitaciones aumentan la posibilidad de gastos de salud catastróficos, ya que la familia igualmente dependerá del sistema público - aunque todavía pague primas de seguro- 0 , si sus inquietudes sobre calidad y acceso son lo suficientemente importantes, pagará directamente la atención como gasto añadido (OckéReis, 2005). Además, hay constancia de que estas limitaciones del seguro son más significativas en las familias pobres, cuyos paquetes de beneficios tienden a ser más restringidos (Bahia, 2001). De hecho, una parte considerable de la población cubierta por el sistema complementario utiliza la red pública, especialmente para procedimientos o tratamientos muy complejos o costosos, como los trasplantes y el tratamiento del VIH/sIDA (Porto, Santos y Ugá, 2006). En estas circunstancias, el seguro privado de salud podría no ser eficaz para reducir los gastos de salud, especialmente si la familia contrató el seguro directamente y tuvo que pagar la totalidad de la prima.

\section{III}

\section{Modelo empírico}

Los gastos de salud se califican de catastróficos si superan cierta proporción del ingreso familiar (el umbral de $40 \%$, por ejemplo). Para estimar el concepto se define la variable binaria CHE (sigla en inglés de gasto sanitario catastrófico) que toma valor uno cuando los gastos de salud superan el umbral y valor cero cuando están por debajo. El modelo empírico es:

$$
\begin{gathered}
\text { Probabilidad }[\mathrm{CHE}=1]=\text { Probabilidad } \\
{[b o+b 1 \cdot \log P+b 2 \cdot \log Y+b 3 . X+u 1>0]}
\end{gathered}
$$

donde $P$ es el precio de la atención de salud, $Y$ es el ingreso y $X$ representa los diversos factores demográficos (sexo, edad, raza y otros), indicadores de seguro de salud e infraestructura de la atención de salud local que podrían afectar el gasto en salud. Con esta ecuación se apunta a estimar cuán importantes son estos factores explicativos para determinar la presencia de gasto de salud catastrófico. Se les estimará a través de una regresión probit.

Si se definiera CHE como una variable binaria sin otros ajustes, el valor cero abarcaría tres grupos: quienes buscaban atención de salud pero para quienes el gasto no superaba el 
umbral pertinente, quienes no recibieron atención de salud aunque la necesitaban, y quienes no tenían necesidad de atención de salud en absoluto. Esta estrategia es a todas luces inapropiada, ya que no distingue entre un resultado favorable — se recibe la atención de salud adecuada (gasto por debajo del umbral) — , uno desfavorable — la atención de salud no se recibe aunque se necesita-, y uno neutral (no se necesita atención de salud). En nuestro análisis se adoptaron dos medidas para evitar esta confusión. En primer lugar, se restringió el análisis a quienes necesitaban atención de salud, como se describe en la sección siguiente. En segundo lugar, se retiró de la estimación probit, mediante el modelo de selección de Heckman, a las personas que no buscaron atención de salud.

Una vez que el análisis queda restringido a quienes sí necesitaban atención de salud, la falta de selección en el modelo de Heckman indica una necesidad no satisfecha: las personas y familias no buscaron atención de salud aunque la necesitaban. Las personas que necesitan atención pero no la obtienen también pueden verse afectadas por un acontecimiento catastrófico (y esto es igualmente una desprotección financiera), pero no están en riesgo de gasto catastrófico (Waters, 2000). El modelo de Heckman de selección de la muestra es un modelo analítico adecuado en este caso (Dow y Norton, 2003), porque está concebido para analizar una situación que tiene un resultado potencial: el de los gastos de salud si la persona hubiera elegido buscar atención. Como es probable que las personas que no buscaban atención (con gastos de salud no observados) sean sistemáticamente diferentes de las personas que sí la buscaban (con gastos de salud observados), es probable que la estimación sufra un sesgo de selección si no se aplica una corrección como el modelo de Heckman (Dow y Norton, 2003).

A continuación se describe la configuración del modelo de selección de Heckman en una estimación probit. La estimación específica del modelo, incluyendo necesidades insatisfechas de atención de salud en Brasil, se presenta más adelante en la sección IV.

$\mathrm{Al}$ igual que en otros modelos probit, se parte del supuesto de que existe una relación subyacente entre una variable continua $y_{j}^{*}$-gastos de salud como fracción del ingreso familiar - y un conjunto de variables explicativas $u_{1 j}$, como se especifica en la siguiente ecuación:

$$
y_{j}^{*}=x_{j} \exists+u_{l j} \quad \text { ecuación latente }
$$

En esta ecuación, $\exists$ es el conjunto de parámetros por estimar y $u_{1 j}$ son los residuos de la estimación. Sin embargo, solamente se mide el resultado binario $\left(y_{j}{ }^{\text {probit }}\right)$ si la variable latente es superior a un umbral dado $(t)$, como se plantea en la siguiente ecuación:

$$
y_{j}{ }^{\text {probit }}=\left(y_{j}^{*}>t\right) \quad \text { ecuación probit }
$$

Sin embargo, la variable dependiente no siempre se observa, ya que los hogares podrían elegir no buscar atención de salud aunque la necesitaran. En lugar de ello, la variable dependiente para observación $j$ se observa si las familias optan por buscar atención de salud, como se especifica en la siguiente ecuación (Van de Ven y Van Pragg, 1981).

$$
y_{j}{ }^{\text {selec }}=\left(z_{j} \vartheta+u_{2 j}>0\right) \quad \text { ecuación de selección }
$$

En la ecuación de selección $y_{j}{ }^{\text {selec }}$ es otra variable binaria $(1=$ hogares que buscaron atención de salud; $0=$ hogares que no la buscaron), $z_{j}$ es un conjunto de variables explicativas de la decisión de selección y $\vartheta$ es el nuevo conjunto de parámetros por estimar. Los residuos de las ecuaciones latente y de selección $\left(u_{l j} \mathrm{y}\right.$ $u_{2 j}$ ) son variables normales, con promedio igual a cero y desviación estándar igual a uno. La correlación entre los residuos, que se espera sea positiva, se mide como rho en las estimaciones que figuran a continuación.

Para estimar el modelo de Heckman se necesitan variables que influyan en la selección — la probabilidad de buscar atención de salud - pero que no afecten directamente la última variable dependiente: la probabilidad de gastos de salud catastróficos (CHE). A estos efectos, se usan tres indicadores del total de la infraestructura de atención de salud a escala regional: cantidad total de unidades ambulatorias, camas de hospital y médicos por habitante. Se trata de variables de identificación - también denominadas variables instrumentales- del modelo, ya que son empleadas en la ecuación de selección de Heckman pero no en la ecuación probit.

Al emplear los procedimientos sugeridos por Murray (2006) se halló que uno de los instrumentos - la cantidad total de unidades ambulatorias per cápita— era a la vez "sólido", es decir, significativo desde el punto de vista estadístico en la ecuación de selección (cuadro 6), y "válido", esto es, no significativo si se incorporaba a la ecuación probit principal. Como se esperaba, esta variable solamente influye en el gasto de atención de salud cuando se busca dicha atención. En cambio, los otros dos instrumentos eran débiles en la mayoría de las definiciones de necesidad de atención de salud. Se efectuó otro análisis — también sugerido por Murray (2006) — mediante la repetición de las estimaciones tras retirar estos instrumentos más débiles. El procedimiento no trajo aparejados cambios importantes en los resultados. Una observación final es que existen correlaciones débiles entre los instrumentos usados en la ecuación de selección y tres variables de control similares usadas en la principal ecuación probit: cantidad de unidades ambulatorias privadas, de camas de hospital y de médicos por habitante. 


\section{IV}

\section{Fuentes de datos y especificaciones empíricas}

\section{Fuentes de datos}

En nuestra investigación se usa un repertorio de datos de la Encuesta nacional de hogares que realiza el Instituto Brasileño de Geografía y Estadística (IBGE). Para la encuesta se recurrió a un proceso de muestreo en tres niveles: municipalidades dentro de cada estado, circunscripciones censales y hogares, sobre la base del censo de 1991 (IBGE, 2000). Los ajustes necesarios para que los resultados fuesen representativos de la población del país en 1998 se basaron en proyecciones de las tasas de natalidad, mortalidad y migración. La muestra abarcó 112.434 hogares y 344.975 personas. Se contrarrestó el efecto de agrupación resultante del diseño de la encuesta mediante la definición de los estados como grupos y el empleo de ponderaciones de probabilidad para cada hogar, sobre la base del conjunto de datos utilizado (StataCorp, 2005).

Una fuente adicional de datos es la Encuesta de atención médica y de salud de 1999 (Ministerio de Salud de Brasil, 2004). La información recogida de esta fuente comprende la infraestructura de atención de salud total y privada, según se detalla a continuación. Dados los niveles de agregación de los resultados informados en el conjunto de datos de la Encuesta nacional de hogares, estos indicadores se usan a nivel de estados y de áreas metropolitanas.

\section{Variables dependientes}

El gasto en salud se calculó mediante la suma de los gastos en los siguientes rubros: medicamentos, primas de seguro - descontado todo aporte del empleador-, visitas médicas, visitas a otros profesionales de la salud, hospitalizaciones, enfermería a domicilio, exámenes, atención odontológica, anteojos y lentes, equipo ortopédico y otros. Como en la encuesta se pregunta sobre gastos directos del hogar, se espera que estos incluyan los costos de los copagos y que consideren los reembolsos de los planes de seguro de salud. Los resultados se ajustan sobre una base mensual, ya que los gastos analizados comprendían a los tres meses anteriores a la encuesta, excepto en el caso de los medicamentos, en que correspondían a un mes. La Encuesta nacional de hogares también entrega información sobre el ingreso mensual de los hogares.
Para la probabilidad de que un hogar tenga gastos de salud catastróficos en un mes específico (ecuación probit), se estimaron tres umbrales alternativos:

i) si el total de gastos de salud es igual o superior al $20 \%$ del ingreso (12\% de los hogares supera este umbral);

ii) si el total de los gastos de salud es igual o superior al $40 \%$ del ingreso (3,7\% de los hogares);

iii) si los gastos de salud excluidas las primas de seguro son equivalentes o superiores al $10 \%$ del ingreso (20\% de los hogares).

Estas estimaciones brutas de gastos de salud catastróficos son muy similares a las conclusiones de Diniz, Servo y otros (2007), quienes usaron datos de otra encuesta, la de presupuestos familiares. La estimación obtenida $(3,7 \%)$ usando el umbral de $40 \%$ es considerablemente menor que la de 10,3\% informada por Xu, Evans y otros (2003); esta diferencia no parece hallar una explicación en las distinciones metodológicas entre los estudios. En Diniz, Servo y otros (2007) se expone en detalle cómo el tamaño de la muestra, la falta de representatividad nacional y los objetivos con que se recopilaron los datos utilizados por Xu, Evans y otros (2003) podría haber llevado a estos autores a sobrestimar la magnitud de los gastos de salud catastróficos en Brasil.

Para la ecuación de selección, la variable dependiente se basa en la pregunta de si algún miembro del hogar buscó atención de salud en las dos semanas previas a la encuesta. Recurriendo a la definición más amplia de la necesidad de atención de salud (la "necesidad de atención de salud $\mathrm{N}^{\circ} 1$ ", según se define más adelante en la sección V), es posible identificar tres grupos de hogares:

i) los que no necesitaron atención de salud (52.504 hogares, es decir, 58\% de la muestra);

ii) los que buscaron atención de salud (32.895 hogares, equivalentes al $36 \%$ de la muestra y al $86 \%$ de los que necesitaron atención), y

iii) los que no buscaron atención de salud aunque la necesitaron (5.514 hogares, que constituyen el $6 \%$ del total de la muestra y el $14 \%$ de los que necesitaron atención).

Las necesidades no satisfechas son un elemento significativo en la encuesta. Uno de cada siete hogares que necesitaron atención de salud no accedió a ella. Entre los hogares que tuvieron necesidades no satisfechas, el $31 \%$ 
hizo presente que el motivo fue la falta de dinero suficiente, el 26\% indicó que tenía problemas con los horarios y el atraso del servicio, el 15\% señaló problemas de acceso, incluidas dificultades de transporte, y el $27 \%$ dio cuenta de otros problemas. Un rasgo interesante que surge de estos datos es que en realidad el no recibir atención luego de buscarla activamente no es un problema generalizado. Solamente el 2\% de los hogares que buscaron atención no la recibieron después de múltiples intentos. Una interpretación de estas conclusiones es que el análisis de Ribeiro, Barata y otros (2006) sobre el poco acceso a la atención de salud en Brasil subestima el problema, ya que se centra en la atención efectivamente recibida, en lugar de centrarse en la decisión de no buscar atención pese a necesitarla. En el mismo sentido, es injustificada la conclusión de la ops (2007, p. 148) de que el acceso a los servicios de salud de la amplia mayoría de la población brasileña parece estar asegurado.

Si bien la unidad de análisis del gasto de salud es el hogar, la unidad de atención de salud es la persona. La agregación del gasto a nivel del hogar es necesaria porque sus integrantes comparten tanto el ingreso como los beneficios de algunos gastos de salud (en particular la prima de seguro).

\section{Necesidad de atención de salud}

Aquí usamos dos definiciones alternativas para la necesidad de atención de salud. La necesidad de atención de salud $\mathbf{N}^{\mathbf{1}} \mathbf{1}$ es la que tienen los hogares donde por lo menos una persona necesitó atención de salud en las dos semanas anteriores a la encuesta, sea cual fuere el motivo. Esta definición abarca el $42 \%$ de los hogares. La necesidad de atención de salud $\mathbf{N}^{\mathbf{2}} \mathbf{2}$ es la que tienen los hogares donde por lo menos una persona adolece de dos o más afecciones crónicas y necesitó atención de salud en las dos semanas anteriores a la encuesta. Esta definición abarca el 18\% de los domicilios.

Habida cuenta de la naturaleza de los datos disponibles, las características del sistema de salud público y los objetivos de este análisis, las regresiones se confinan a las personas y los hogares que necesitaron atención de salud. Mediante regresiones logísticas sencillas en cada una de estas definiciones de atención de salud se indica la forma en que ellas restringen la muestra. Por limitaciones de espacio no se incorporan aquí relaciones detalladas de estos análisis, sino que se resumen los principales resultados. Con la definición $\mathrm{N}^{\circ}$ 1, la necesidad es más probable si el hogar tiene mayor porcentaje de mujeres, si incluye por lo menos un adulto mayor, si tiene un mayor número de integrantes y si se encuentra en zonas urbanas. Los hogares más pobres tienen igual probabilidad de necesitar atención de salud que los más acomodados.

Con la definición $\mathrm{N}^{\mathrm{o}} 2$ los resultados son similares, siendo más acentuada la importancia de la participación femenina y la presencia de personas mayores, y teniendo los hogares más pobres mayor necesidad de atención de salud que los más acomodados. Nótese que no hay un gradiente de necesidad de salud vinculado al ingreso en la definición más amplia de necesidad de salud (la $\mathrm{N}^{\mathrm{o}} 1$ ), pero que sí lo hay en la definición que incorpora las afecciones crónicas (la $\mathrm{N}^{\circ} 2$ ). Estas conclusiones sugieren que la mayor necesidad de atención de salud en hogares más pobres está asociada a la predominancia de afecciones crónicas en este grupo, como lo señalan Almeida, Barata y otros (2002).

En la encuesta se registró la necesidad de atención de salud en las dos semanas previas, mientras que la información sobre gastos de salud se recopiló para estimar los gastos catastróficos en los tres meses anteriores (un mes en el caso de medicamentos). Esta diferencia de plazos podría introducir un sesgo hacia personas y hogares que necesitan atención de salud más frecuente y que, por lo tanto, tienen más probabilidades de ser captados por un muestreo de dos semanas. En este caso, el sesgo es idéntico al que se introduce al restringir el análisis a quienes necesitaron asistencia sanitaria.

\section{Variables explicativas de interés}

Las variables primarias de interés son:

i) Si el hogar usó el sistema único de salud pública de Brasil para atender sus necesidades de salud en las dos semanas anteriores a la encuesta (sí $=44 \%$ de los hogares que buscaron atención de salud). Esta variable no fue usada en la ecuación de selección.

ii) Si alguien del hogar tiene seguro privado de salud (33,5\% de los hogares).

iii) Logaritmo del ingreso mensual familiar, en reales. El uso del sus y la afiliación al seguro privado de salud no son recíprocamente excluyentes, ya que la afiliación al seguro privado no impide que se utilice el sistema público. Pero de los hogares que recibieron atención de salud y tenían seguro, solamente el $21 \%$ usó el sus. Esta superposición entre el sistema público y el seguro privado podría afectar los resultados de estas dos variables. Sin embargo, las repeticiones para evaluar el efecto de la superposición demuestran que esta no afecta nuestros resultados de manera importante. Específicamente, las regresiones se estimaron mediante el retiro de una de las dos variables y la verificación de cómo se afecta la otra variable, y se realizaron nuevas regresiones con 
indicadores de interacción entre ellas. Por consideraciones de brevedad los resultados no se presentan.

El seguro privado de salud podría ser endógeno al estado de salud, ya que quienes tienen peor estado de salud podrían buscar la obtención de un seguro (selección adversa). Los datos con los que se cuenta no permiten descartar esta posibilidad, pero no parece probable. Por ejemplo, hay poca relación entre la cantidad de afecciones crónicas y el seguro de salud, ya que el $26 \%$ de las personas sin afecciones crónicas, el $24 \%$ de quienes tienen una afección crónica y el $22 \%$ de quienes tienen dos afecciones crónicas cuentan con seguro. En igual sentido, la cobertura del seguro privado es más alta $(25,9 \%)$ entre quienes evalúan su propia salud como excelente o buena y menor $(14,5 \%)$ entre quienes evalúan su salud como mala o muy mala (IBGE, 2000). Este patrón parece ser compatible con la selección por riesgo de las aseguradoras pero incompatible con la selección adversa sencilla. En un análisis más riguroso de los mismos datos usados en esta investigación, Bahia, Costa y otros (2002) llegaron a la conclusión de que la selección adversa no era importante.
Como cabía esperar, los hogares más pobres tienen más probabilidad de depender del sistema de salud pública y menos probabilidad de contar con seguro privado. Si se divide la muestra en mitades, a partir del ingreso per cápita del hogar, el 70\% de los hogares más pobres que recibieron atención de salud en las dos semanas anteriores usaron el sus, mientras que el $36 \%$ de los hogares más ricos usaron el sistema público. El seguro cubre solo el $13 \%$ de los domicilios más pobres y el $55 \%$ de los más ricos. Estas relaciones indican la importancia de considerar el ingreso cuando se analiza el efecto del sistema público y del seguro privado en el nivel de los gastos de salud. Se reprodujeron todas las regresiones en submuestras de los más pobres y los más ricos. En aras de la brevedad no se presenta una relación detallada de estas repeticiones. En general, ellas confirman las principales conclusiones del análisis de la muestra completa.

\section{Variables de control}

En el cuadro 5 se describen las variables de control.

CUADRO 5

Brasil: variables de control

\begin{tabular}{|c|c|c|}
\hline \multirow{3}{*}{$\begin{array}{l}\text { Infraestructura de atención } \\
\text { de salud privada a nivel } \\
\text { estadual y metropolitano } \\
\text { (solo para ecuación probit) }\end{array}$} & Cantidad de unidades ambulatorias que no atienden el sus/1000 personas & $\begin{array}{l}\text { promedio: } 0,06 ; \\
\text { rango: } 0,01 \text { a } 0,22\end{array}$ \\
\hline & Cantidad de camas de hospital que no atienden el sus/1000 personas & $\begin{array}{l}\text { promedio: } 0,38 ; \\
\text { rango: } 0,02 \text { a } 0,87\end{array}$ \\
\hline & Cantidad de médicos que no atienden el sus/1000 personas & $\begin{array}{l}\text { promedio: } 1,3 ; \\
\text { rango: } 0,08 \text { a } 2,79\end{array}$ \\
\hline \multirow{8}{*}{ Rasgos demográficos } & Sexo: porcentaje de mujeres en el hogar & promedio: 51,7 \\
\hline & Raza: si el jefe/jefa de familia es mulato/mulata (\% de la muestra) & 39 \\
\hline & Raza: a si el jefe/jefa de familia es negro/ negra (\% de la muestra) & 6,8 \\
\hline & Niños: si hay en el hogar por lo menos un niño o niña de 10 años o menos (\% de la muestra) & 44 \\
\hline & Personas mayores: si hay en el hogar por lo menos una persona de 65 años o más (\% de la muestra) & 17,4 \\
\hline & Educación: nivel educativo más alto en el hogar, medido en años de escolaridad & Promedio $=7,8$ años \\
\hline & Tamaño del hogar (\% de la muestra) & $\begin{array}{l}1 \text { persona }=9 \% \\
2 \text { personas }=17 \% \\
3 \text { a } 5 \text { personas }=60 \% \\
6 \text { o más }=14 \%\end{array}$ \\
\hline & Si el domicilio es en una región rural (\% de la muestra) & 16 \\
\hline \multirow{2}{*}{$\begin{array}{l}\text { Precios de la atención de } \\
\text { salud a nivel estadual y } \\
\text { metropolitano }\end{array}$} & Costo medio de hospitalización según está aprobado por el sus & $\begin{array}{l}\text { Promedio: } 357 \text { reales; } \\
\text { Rango: } 207 \text { a } 566 \text { reales }\end{array}$ \\
\hline & Valor medio de un procedimiento ambulatorio según está aprobado por el sus & $\begin{array}{l}\text { Promedio: } 3,3 \text { reales; } \\
\text { Rango: } 2,0 \text { a 4,4 reales }\end{array}$ \\
\hline \multirow{3}{*}{$\begin{array}{l}\text { Infraestructura total de } \\
\text { atención de salud a nivel } \\
\text { estadual y metropolitano } \\
\text { (solo para la ecuación de } \\
\text { selección) }\end{array}$} & Cantidad total de unidades ambulatorias/1000 personas & $\begin{array}{l}\text { Promedio: } 0,24 ; \\
\text { Rango: } 0,11 \text { a 0,61 }\end{array}$ \\
\hline & Cantidad total de camas de hospital/1000 personas & $\begin{array}{l}\text { promedio: } 3,0 ; \\
\text { rango: } 1,8 \text { a } 4,0\end{array}$ \\
\hline & Cantidad total de médicos/1000 personas & $\begin{array}{l}\text { promedio: } 4,3 ; \\
\text { rango: } 0,8 \text { a } 6,7\end{array}$ \\
\hline
\end{tabular}

Fuente: IBGE (2000) y Ministerio de Salud de Brasil (2004).

a La categoría de raza es básicamente blanca (53,5\% de la muestra); otras razas serían solamente $0,7 \%$ de la muestra. 
V

\section{Resultados y análisis}

En el cuadro 6 se muestra tanto el resultado de la ecuación probit como el de la ecuación de selección. En la ecuación probit se presentan efectos marginales para la probabilidad de que un hogar enfrente gastos de salud catastróficos si busca atención de salud. En la ecuación de selección se muestra el efecto marginal para la probabilidad de que un hogar busque atención de salud. En ambos casos, para las variables independientes

CUADRO 6

Brasil: resultado de las regresiones

\begin{tabular}{|c|c|c|c|c|c|c|}
\hline & \multicolumn{3}{|c|}{ Necesidad de asistencia sanitaria $\mathrm{N}^{\circ} 1$ : } & \multicolumn{3}{|c|}{ Necesidad de asistencia sanitaria $\mathrm{N}^{\circ} 2$ : } \\
\hline & $\begin{array}{c}\text { Gastos de salud } \\
\text { catastróficos } \\
20 \%\end{array}$ & $\begin{array}{c}\text { Gastos de salud } \\
\text { catastróficos } \\
40 \%\end{array}$ & $\begin{array}{l}\text { Gastos de salud } \\
\text { catastróficos, } \\
\text { del bolsillo }\end{array}$ & $\begin{array}{c}\text { Gastos de salud } \\
\text { catastróficos } \\
20 \%\end{array}$ & $\begin{array}{c}\text { Gastos de salud } \\
\text { catastróficos } \\
40 \%\end{array}$ & $\begin{array}{l}\text { Gastos de salud } \\
\text { catastróficos, } \\
\text { del bolsillo }\end{array}$ \\
\hline Ecuación probit ${ }^{\mathrm{a}}$ & $y=0,232$ & $y=0,074$ & $y=0,378$ & $y=0,347$ & $y=0,142$ & $y=0,546$ \\
\hline Ingreso (logaritmo) & $-0,135^{\mathrm{b}}$ & $-0,077^{\mathrm{b}}$ & $-0,127^{b}$ & $-0,168^{b}$ & $-0,118^{\mathrm{b}}$ & $-0,136^{\mathrm{b}}$ \\
\hline Si usó el sus & $-0,063^{\mathrm{b}}$ & $-0,035^{\mathrm{b}}$ & $-0,041^{\mathrm{b}}$ & $-0,094^{b}$ & $-0,058^{\mathrm{b}}$ & $-0,052^{b}$ \\
\hline Con seguro de salud & $0,096^{\mathrm{b}}$ & $0,027^{b}$ & $-0,004$ & $0,140^{\mathrm{b}}$ & $0,053^{\mathrm{b}}$ & 0,001 \\
\hline Ambulatorio privado & $0,204^{\mathrm{d}}$ & 0,045 & $-0,089$ & 0,385 & $-0,047$ & 0,137 \\
\hline Camas de hospital privado & $0,100^{\mathrm{b}}$ & $0,042^{\mathrm{c}}$ & $0,082^{b}$ & 0,106 & 0,034 & 0,047 \\
\hline Médicos privados & $-0,028^{\mathrm{c}}$ & $-0,012$ & $-0,010$ & $-0,030$ & $-0,007$ & 0,003 \\
\hline$\%$ de mujeres & $0,074^{\mathrm{b}}$ & $0,027^{\mathrm{b}}$ & $0,072^{b}$ & $0,078^{\mathrm{b}}$ & $0,031^{\mathrm{d}}$ & $0,057^{\mathrm{b}}$ \\
\hline Raza: mulata & $-0,052^{b}$ & $-0,029^{\mathrm{b}}$ & $-0,050^{\mathrm{b}}$ & $-0,071^{b}$ & $-0,042^{b}$ & $-0,065^{b}$ \\
\hline Raza: negra & $-0,057^{b}$ & $-0,032^{\mathrm{b}}$ & $-0,048^{\mathrm{b}}$ & $-0,094^{b}$ & $-0,057^{\mathrm{b}}$ & $-0,080^{\mathrm{b}}$ \\
\hline Con niños & $-0,076^{\mathrm{b}}$ & $-0,041^{\mathrm{b}}$ & $-0,111^{\mathrm{b}}$ & $-0,041^{\mathrm{b}}$ & $-0,040^{\mathrm{b}}$ & $-0,054^{\mathrm{b}}$ \\
\hline Con adultos mayores & $0,134^{\mathrm{b}}$ & $0,063^{\mathrm{b}}$ & $0,158^{\mathrm{b}}$ & $0,096^{\mathrm{b}}$ & $0,059^{\mathrm{b}}$ & $0,099^{\mathrm{b}}$ \\
\hline Nivel educativo más alto en el hogar & 0,002 & 0,001 & 0,000 & 0,002 & $0,003^{\mathrm{c}}$ & 0,002 \\
\hline Tamaño del hogar & $-0,005^{\mathrm{b}}$ & $-0,001$ & $-0,003$ & $-0,008^{\mathrm{b}}$ & $-0,002$ & $-0,007^{\mathrm{b}}$ \\
\hline Si se halla en zonas rurales & 0,011 & 0,003 & 0,015 & $-0,006$ & 0,003 & 0,002 \\
\hline Costo de hospital $(\log )$ & $-0,073^{b}$ & $-0,009$ & $-0,109^{\mathrm{b}}$ & $-0,100$ & $-0,034$ & $-0,180^{\mathrm{c}}$ \\
\hline Costo ambulatorio (log) & $0,099^{\mathrm{b}}$ & 0,018 & $0,104^{\mathrm{b}}$ & $0,186^{\mathrm{b}}$ & 0,047 & $0,202^{\mathrm{b}}$ \\
\hline Ecuación de selección & año $=0,886$ & año $=0,886$ & año $=0,887$ & año $=0,7970$ & año $=0,797$ & año $=0,797$ \\
\hline Ingreso (log) & $0,032^{\mathrm{b}}$ & $0,031^{\mathrm{b}}$ & $0,034^{\mathrm{b}}$ & $0,046^{\mathrm{b}}$ & $0,045^{\mathrm{b}}$ & $0,049^{\mathrm{b}}$ \\
\hline Con seguro de salud & $0,047^{\mathrm{b}}$ & $0,047^{\mathrm{b}}$ & $0,046^{\mathrm{b}}$ & $0,064^{\mathrm{b}}$ & $0,064^{\mathrm{b}}$ & $0,063^{b}$ \\
\hline Total ambulatorios & $0,207^{\mathrm{b}}$ & $0,213^{\mathrm{b}}$ & $0,195^{\mathrm{b}}$ & $0,213^{\mathrm{c}}$ & $0,218^{c}$ & $0,205^{\mathrm{d}}$ \\
\hline Total de camas de hospital & $-0,006$ & $-0,008$ & $-0,004$ & $-0,002$ & $-0,004$ & 0,001 \\
\hline Total de médicos & $0,008^{\mathrm{c}}$ & $0,008^{\mathrm{c}}$ & $0,007^{\mathrm{b}}$ & 0,010 & 0,010 & 0,010 \\
\hline Porcentaje femenino & 0,004 & 0,004 & 0,003 & 0,011 & 0,011 & 0,011 \\
\hline Raza: mulato & $-0,025^{\mathrm{b}}$ & $-0,025^{\mathrm{b}}$ & $-0,025^{\mathrm{b}}$ & $-0,035^{\mathrm{b}}$ & $-0,035^{\mathrm{b}}$ & $-0,035^{\mathrm{b}}$ \\
\hline Raza: negra & $-0,028^{\mathrm{b}}$ & $-0,028^{\mathrm{b}}$ & $-0,028^{\mathrm{b}}$ & $-0,012$ & $-0,012$ & $-0,013$ \\
\hline Con niños & $0,041^{\mathrm{b}}$ & $0,041^{\mathrm{b}}$ & $0,042^{\mathrm{b}}$ & $-0,011$ & $-0,011$ & $-0,010$ \\
\hline Con adultos mayores & $-0,015^{\mathrm{b}}$ & $-0,014^{b}$ & $-0,016^{\mathrm{b}}$ & 0,009 & 0,008 & 0,008 \\
\hline Nivel educativo más alto en el hogar & $0,006^{\mathrm{b}}$ & $0,006^{\mathrm{b}}$ & $0,006^{\mathrm{b}}$ & $0,006^{\mathrm{b}}$ & $0,005^{\mathrm{b}}$ & $0,005^{b}$ \\
\hline Tamaño del hogar & $-0,001$ & $-0,001$ & $-0,001$ & $-0,002$ & $-0,002$ & $-0,002$ \\
\hline Si se halla en zonas rurales & $-0,043^{b}$ & $-0,043^{b}$ & $-0,042^{\mathrm{b}}$ & $-0,073^{b}$ & $-0,073^{b}$ & $-0,072^{b}$ \\
\hline Costo de hospital (log) & 0,049 & $0,052^{\mathrm{d}}$ & $0,049^{\mathrm{b}}$ & 0,077 & 0,080 & 0,074 \\
\hline Costo de servicios ambulatorios (log) & $0,114^{\mathrm{b}}$ & $0,113^{\mathrm{b}}$ & $0,116^{\mathrm{b}}$ & $0,213^{\mathrm{b}}$ & $0,167^{b}$ & $0,170^{b}$ \\
\hline Estadístico rho & $-0,625^{\mathrm{b}}$ & $-0,554^{\mathrm{b}}$ & $-0,804^{\mathrm{b}}$ & $-0,484^{\mathrm{b}}$ & $-0,530^{\mathrm{b}}$ & $-0,704^{\mathrm{b}}$ \\
\hline Observaciones & 36738 & 36738 & 36738 & 15317 & 15317 & 15317 \\
\hline Estadística F & $137,38^{b}$ & $102,07^{\mathrm{b}}$ & $169,38^{b}$ & $52,24^{\mathrm{b}}$ & $63,07^{\mathrm{b}}$ & $51,41^{\mathrm{b}}$ \\
\hline
\end{tabular}

Fuente: IBGE (2000) y Ministerio de Salud de Brasil (2004).

a " $\mathrm{y}=$ " indica la probabilidad del resultado

b Significativo en el $1 \%$

c Significativo en el 5\%

d Significativo en el $10 \%$ 
continuas, los efectos marginales muestran cómo cambia la variable dependiente a medida que la independiente varía en forma infinitesimal y se calculan en la media de la variable independiente. En las variables binarias se calcularon los efectos como el cambio discreto de la variable dependiente cuando la variable independiente pasa de 0 a 1 . Las pruebas de significación estadística se basaron en estimadores de varianza robustos de Huber-White.

\section{Análisis de selección}

Los hogares de ingresos más altos tienen mayor probabilidad de recibir atención de salud, conclusión similar a los de otros estudios brasileños (Bahia, Costa y otros, 2002; Farias y Melamed, 2003; Mendoza-Sassi, Béria y Barros, 2003; Neri y Soares, 2002). Los hogares con seguro de salud privado tienen probabilidad entre el $5 \%$ y el $8 \%$ superior de recibir atención de salud, resultado similar al obtenido por Pinheiro y Travassos (1999), Mendoza-Sassi, Béria y Barros (2003), Neri y Soares (2002) y Viacava, Souza-Júnior y Szwarcwald (2005). Incluso en el contexto de un sistema único de salud gratuito, el acceso a la atención de salud está limitado por el ingreso del hogar y el seguro de salud. Los hogares de menores ingresos sin seguro privado tienen mayor probabilidad de que algunas de sus necesidades de atención de salud no sean satisfechas.

\section{Factores determinantes de gastos de salud catastróficos (ecuación probit)}

Como era de esperar, el ingreso tiene un efecto importante, ya que los hogares más pobres que buscan atención de salud tienden a gastar más en atención de salud como porcentaje de sus ingresos. El uso del sus disminuye en $47 \%$ la probabilidad de que el hogar enfrente gastos de salud catastróficos $(0,035 / 0,074$, en el umbral de $40 \%$ y con la necesidad de atención de salud $\mathrm{N}^{\circ} 1$ ). Como esta variable solo se refiere a dos semanas, el efecto en un mes completo sería superior.

En el cuadro 7 se comparan los gastos de atención de salud como porcentaje del gasto total para distintos umbrales. El gasto más importante es en medicamentos, seguido por las primas de seguro de salud —obviamente, no en el umbral del gasto de bolsillo (out-of-pocket expenditure)-, atención odontológica, hospitalización y visitas médicas. Cabe destacar el mayor peso de las hospitalizaciones en el umbral del 40\%; si algún integrante del hogar es internado, aumenta considerablemente la probabilidad de que se cruce este umbral.

¿Por qué los hogares necesitan gastar dinero cuando recurren al sus? Una respuesta posible es que algún miembro del hogar no haya usado el sus, mientras que otros sí lo hayan hecho. La segmentación del mercado de seguro de salud privado (Pinto y Soranz, 2004; Bahia, 2001) que cubre primordialmente a hogares de mayores ingresos que tienen menor probabilidad de usar el sus, sugiere que tal situación no es muy frecuente. Otro punto es que en los domicilios que usaron el sus y tenían necesidad de atención de salud (necesidad $\mathrm{N}^{\circ} 1$ ), el 66\% de los gastos medios en salud guardan relación con medicamentos, lo cual indica que la cobertura del sus es especialmente débil en esta categoría (Lóbrega, Marques y otros, 2007). El sus solamente despacha medicamentos en farmacias y unidades ambulatorias públicas. Si un paciente, con receta de un médico del sus, no puede obtener el medicamento en un servicio público, tiene que pagarlo en una farmacia privada. Esta transferencia de gastos - del proveedor público al paciente individual- es una fuente importante de los gastos de salud del hogar. Este problema también fue identificado por Silveira, Osório y Piola (2002),

CUADRO 7

Brasil: gastos de atención de salud

(Como porcentaje del gasto total en salud)

\begin{tabular}{lccc}
\hline Gastos & $\begin{array}{c}\text { Atención de salud 20\% } \\
\text { (en porcentajes) }\end{array}$ & $\begin{array}{c}\text { Atención de salud 40\% } \\
\text { (en porcentajes) }\end{array}$ & $\begin{array}{c}\text { Atención de salud, gasto } \\
\text { del bolsillo (en porcentajes) }\end{array}$ \\
\hline Medicamentos & 41,6 & 42,3 & 53,6 \\
Prima de seguro & 20,9 & 13,3 & $\ldots$ \\
Atención odontológica & 13,9 & 13,1 & 18,1 \\
Hospitalización & 7,5 & 13,5 & 7,5 \\
Visitas médicas & 4,6 & 5,6 & 6,0 \\
Anteojos y lentes & 3,9 & 2,9 & 5,5 \\
Análisis & 3,2 & 4,0 & 3,9 \\
Otro & 4,5 & 5,2 & 5,3 \\
\hline
\end{tabular}

Fuente: IBGE (2000). 
Lima-Costa, Barreto y Giatti (2003) y Arrais, Luciara $y$ otros (2005).

El seguro privado de salud está asociado a un $36 \%$ de aumento en la probabilidad de gastos de salud catastróficos $(0,027 / 0,074$, en el umbral del $40 \%$ y con la necesidad de atención de salud $\mathrm{N}^{\mathrm{o}} 1$ ). Este resultado parece contrario al sentido común, ya que se supone que el seguro es una protección (Sekhri y Savedoff, 2005). El seguro privado no ha sido eficaz en reducir la carga financiera del hogar asociada a la atención de salud, ya que los costos financieros de las primas eran más importantes que la reducción esperada de los gastos financieros cuando se recurrió a la atención privada. En realidad, como muestra el cuadro 6, la variable seguro privado de salud no es significativa en la regresión probit en el caso del umbral de los gastos de bolsillo. Este hallazgo indica que el seguro de salud no tiene efectos en los gastos de bolsillo a nivel del hogar. El seguro está asociado a aumentos considerables en los gastos de atención de salud en todas las categorías. Parte de este aumento se explica por el mayor uso de esa atención, pero incluso si se restringe el cálculo a los hogares que recurrieron a atención de salud en los últimos 14 días, el gasto medio total de quienes tienen seguro privado casi cuadruplica el gasto de quienes no lo tienen (251 reales contra 63 reales). Del aumento, el $44 \%$ se debe a la prima media de atención de salud.

La importancia del costo de la prima de seguro en el presupuesto total de atención de salud también fue señalada por Silveira, Osório y Piola (2002) y por Kilsztajn, Camara y Carmo (2002). Los resultados también son compatibles con la observación de Viacaba, Souza-Júnior y Szwarcwald (2005) de que las personas con planes privados de salud no siempre usan su seguro

\section{VI}

\section{Conclusiones}

El sistema de salud pública brasileño aporta una importante reducción (47\%) de la probabilidad de que un hogar tenga niveles catastróficos de gastos de salud. Según Ribeiro, Barata y otros (2006), este beneficio es más importante para los hogares pobres, que tienden a depender más del sistema público. Otros autores, entre ellos Ocké-Reis, Silveira y Andreazzi (2002), Diaz (2003) y Porto, Santos y Ugá (2006), han señalado el efecto protector del sus. La alta carga financiera inhe- para pagar los servicios. Farias (2001) explica la decisión de contratar un seguro de salud privado por cuatro elementos: calidad, acceso, confiabilidad y estatus social. Los prestadores públicos son considerados de mala calidad; como los médicos tienen tiempo y recursos limitados para cada consulta, hay que esperar más para consultar y no hay garantía de que la consulta a través del sus vaya a ser posible en un momento determinado. Farias sugiere asimismo que usar un proveedor privado podría ser visto como una estrategia para mantener el estatus social. Según datos de la Encuesta nacional de hogares, el $80 \%$ de los planes de seguro ofrecen servicios a través de sus propias redes de atención y el $88 \%$ tiene una red de profesionales privados contratados. Está claro que los médicos, hospitales, laboratorios y otros servicios del primer grupo (red propia) no están disponibles para personas que no tienen seguro de salud y lo mismo ocurre en gran medida con los del segundo grupo (red contratada).

La motivación para contratar un seguro privado es el acceso a servicios de mejor calidad y más confiables - como lo indican los resultados de la ecuación regresiva de selección - y no necesariamente la reducción de los gastos de bolsillo, como muestran los resultados de la ecuación probit regresiva. De forma similar, otra motivación puede deberse a distinciones dentro del hogar, si el seguro no cubre a todos sus integrantes. Si las personas que no tienen seguro igualmente evitan usar el sus - habida cuenta de las inquietudes sobre acceso, calidad y estatus social- tendrán que soportar toda la carga financiera que significan los proveedores privados. Esto es muy importante ya que en el $40 \%$ de los hogares con alguna cobertura de seguro de salud hay por lo menos una persona no cubierta por ese seguro.

rente a la atención de salud en Brasil, en comparación con otros países, puede atribuirse a los aspectos de esa atención que no están bien cubiertos por el sistema público - en especial los medicamentos con y sin receta médica-y las deficiencias en cantidad, accesibilidad, calidad y confiabilidad de los prestadores públicos, que llevan a muchos hogares a recurrir al sector privado, inclusive la contratación de un seguro privado de salud (Bós, 2007). 
En Brasil, para una amplia mayoría de la población, el seguro privado de salud no da protección eficaz desde el punto de vista financiero y no llega a los hogares más pobres. Si bien es una herramienta para obtener acceso a proveedores privados, no reduce los gastos de bolsillo del hogar y las primas son relativamente caras. De lo dicho se desprende entonces que el seguro privado de salud está categóricamente asociado a gastos de salud catastróficos.

Desde 1998, los servicios de salud prestados conforme a planes privados han estado sometidos a reglamentaciones, normas, controles y fiscalización de la Agencia Nacional de Salud Complementaria. Esta nueva reglamentación se tradujo en algunos beneficios preceptivos, pero elevó considerablemente los costos de la prima y sus efectos son limitados (Farias y Melamed, 2003; Ocké-Reis, 2005; Fernández, Pires y otros, 2007). Para los fines de nuestra investigación, puede entenderse que las nuevas reglamentaciones no apuntan a ampliar la protección financiera de los clientes del seguro de salud, sino a minimizar la práctica de selección de riesgo de las aseguradoras (Malta, Cecílio y otros, 2004). Por lo tanto, se espera que los resultados exhibidos no hayan cambiado significativamente desde entonces. Sin ir más lejos, Ocké-Reis y Cardoso (2006) encontraron que si bien la Agencia Nacional de Salud Complementaria reglamenta los precios que cobran algunos planes privados de salud, en el período 2001-2005 estos precios aumentaron más rápidamente que la inflación del sector de la salud. En el mismo sentido, Diniz, Servo y otros (2007) informan que el gasto medio, ajustado por inflación, de los hogares con primas de planes de salud era superior en el 2003 que en 1996, en tanto que Andrade y Maia (2007) hallaron muy pocas diferencias en los factores determinantes de la demanda de seguro privado de salud entre 1998 y el 2003.

Las recomendaciones encaminadas a perfeccionar el sistema único de salud de Brasil apuntan a mayores mejoras en la cantidad, accesibilidad, calidad y confiabilidad de los prestadores públicos. En la medida en que el sistema de atención de salud mejore, los hogares lo elegirán y menos de ellos estarán sometidos a gastos de salud catastróficos. Nuestra investigación halló, además, que la búsqueda de atención de salud está limitada por la infraestructura de esa atención en el ámbito regional, especialmente a nivel de atención primaria. Más inversión en la cantidad de prestadores de atención primaria de la salud - tanto pública como privada - ampliará el acceso y reducirá la probabilidad de necesidades de atención insatisfechas. Mejorar la red pública tendrá el doble beneficio de dar acceso a los servicios de salud y de mejorar la protección financiera en el lugar de atención. En cuanto a efectos directos sobre el gasto de salud, queda claro que debería hacerse hincapié en una provisión más amplia y adecuada de medicamentos con y sin receta. El impulso que se está dando últimamente a la oferta de fármacos genéricos en farmacias públicas y privadas es beneficioso, porque baja los costos del sistema público en las primeras y los costos para los pacientes en las segundas. Sin embargo, el efecto de esta tendencia es aún limitado. Bertoldi, Barros y Hallal (2005) estimaron que los fármacos genéricos eran solamente el $4 \%$ del total de medicamentos usados. Es necesario un empeño mayor para mejorar el acceso a medicamentos, pese a los altos costos que esto acarrearía para el sistema público y a las complejidades de establecer un sistema de fiscalización eficaz. Existe incongruencia entre la forma en que el sus presta la mayoría de los servicios de salud asistencia sanitaria —además de usar prestadores públicos reembolsa a prestadores privados por sus servicios- $\mathrm{y}$ la forma en que entrega medicamentos, los que solo están disponibles en farmacias y dispensarios públicos. En algún momento futuro el sus debería comenzar a reembolsar a las farmacias privadas por dispensar medicamentos a sus pacientes.
Almeida, M.F., R.B. Barata y otros (2002): Prevalence of self reported chronic diseases and health services consumption from the National Household Sample Survey of 1998 in Brazil, Ciência \& saúde coletiva, vol. 7, $\mathrm{N}^{\circ}$ 4, Rio de Janeiro, Associação Brasileira de Pós-Graduação em Saúde Coletiva.

Alves, D. y Ch. Timmins (2003): Social exclusion and the two-tiered healthcare system of Brazil, en J. Behrman, A. Gaviria y M. Szekely (comps.), Who's In and Who's Out: Social Exclusion in Latin America, Washington, D.C., Banco Interamericano de Desarrollo.

Andrade, M.V. y A.C. Maia (2007): Demanda por planos de saúde no Brasil, en F.G. Silveira, L.M. Servo y otros (comps.),
Gasto e consumo das famílias brasileiras contemporâneas, vol. 2, Brasilia, Instituto de Investigación Económica Aplicada (IPEA).

Arrais, P.S., L.B. Luciara y otros (2005): Prevalence and determinants of medicines consumption in Fortaleza, Ceará, Brazil, Cadernos de saúde pública, vol. 21, № 6, Rio de Janeiro, Escola Nacional de Saúde Pública.

Bahia, L. (2001): Private health plans: light and shadow in the 1990s health sector debate, Ciência \& saúde coletiva, vol. $6, \mathrm{~N}^{\circ} 2$, Rio de Janeiro, Associação Brasileira de Pós-Graduação em Saúde Coletiva. 
Bahia, L., A.J.L. Costa y otros (2002): Segmentation of the demand of the plans and private insurances of health: an analysis of the information of PNAD/98, Ciência \& saúde coletiva, vol. 7, $N^{\circ}$ 4, Rio de Janeiro, Associação Brasileira de Pós-Graduação em Saúde Coletiva.

Banco Mundial (2001): World Development Report 2001: Attacking Poverty, Washington, D.C., Oxford University Press.

Bertoldi, A.D., A.J.D. Barros y P.C. Hallal (2005): Generic drugs in Brazil: known by many, used by few, Cadernos de saúde pública, vol. 21, $\mathrm{N}^{\mathrm{o}} 6$, Rio de Janeiro, Escola Nacional de Saúde Pública.

Bós, A.M. (2007): Health care provider choice among the elderly in a state in Brazil: a structural model, Revista Panamericana de Salud Pública, vol. 22, № 1, Washington, D.C., Organización Panamericana de la Salud.

Bós, A.M. y A.J. Bós (2004): Determinants of elders' choice between private and public health care providers, Revista de Saúde Pública, vol. 38, No 1, São Paulo, Facultad de Salud Pública, Universidad de São Paulo.

Cornwall, A. y A. Shankland (2008): Engaging citizens: lessons from building Brazil's national health system, Social Science \& Medicine, vol. 66, $\mathrm{N}^{\circ} 10$, Amsterdam, Elsevier.

Costa, J. y J. García (2003): Demand for private health insurance: how important is the quality gap?, Health Economics, vol. 12, $\mathrm{N}^{\mathrm{o}} 7$, Nueva York, Wiley \& Sons.

De Abreu, D.M.X., C.C. César y E.B. França (2007): The relationship between deaths that are avoidable with adequate health care and the implementation of the Unified Health System in Brazil, Revista Panamericana de Salud Pública, vol. 21, № 5, Washington, D.C., Organización Panamericana de la Salud.

Diaz, M.D.M. (2003): Desigualdades socieconômicas na saúde, Revista brasileira de economia, vol. 57, № 1, Rio de Janeiro, Fundación Getúlio Vargas.

Diniz, B.P.C., L.M.S. Servo y otros (2007): Gasto das famílias com saúde no Brasil: evolução e debate sobre gasto catastrófico, en F.G. Silveira, L.M. Servo y otros (comps.), Gasto e consumo das famílias brasileiras contemporâneas, vol. 2, Brasilia, Instituto de Investigación Económica Aplicada.

Dow, W.H. y E.C. Norton (2003): Choosing between and interpreting the Heckit and two-part models for corner solutions, Health Services \& Outcomes Research Methodology, vol. 4, Springer.

Escorel, S., L. Giovanella y otros (2007): O Programa de Saúde da Família e a construção de um novo modelo para a atenção básica no Brasil, Revista Panamericana de Salud Pública, vol. 21, N² 2, Washington, D.C., Organización Panamericana de la Salud.

Farias, L.O. (2001): Individual health protection strategies: a study on adherence to the supplementary health system, Ciência \& saúde coletiva, vol. $6, \mathrm{~N}^{\circ} 2$, Rio de Janeiro, Associação Brasileira de Pós-Graduação em Saúde Coletiva.

Farias, L.O. y C. Melamed (2003): Market shares in the Brazilian health sector, Ciência \& saúde coletiva, vol. 8, № 2, Rio de Janeiro, Associação Brasileira de Pós-Graduação em Saúde Coletiva.

Fernández. E., H.M. Pires y otros (2007): An analysis of the supplementary health sector in Brazil, Health Policy, vol. 81, $\mathrm{N}^{\mathrm{o}} 2-3$, Amsterdam, Elsevier.

Gershman, S. y M.A.B. Santos (2006): The Brazilian Public Health System (Sistema Único de Saúde) as the unfolding of twentieth century health policies, Revista brasileira de ciências sociais, vol. 21, N ${ }^{\circ}$ 61, São Paulo, Associação Nacional de Pós-Graduação e Pesquisa em Ciências Sociais.

Gertler, P. y J. Gruber (2002): Insuring consumption against illness, American Economic Review, vol. 92, № 1, Nashville, Tennessee, American Economic Association.

Hulme, D. y A. Shepherd (2003): Conceptualizing chronic poverty, World Development, vol. 31, № 3, Amsterdam, Elsevier.

IBGE (Instituto Brasileño de Geografía y Estadística) (2000): Pesquisa Nacional por Amostra de Domicílios: acesso e utilização de serviços de saúde - 1998, Rio de Janeiro.
(2006): Tábulas completas de mortalidade - 2006, Río de Janeiro.

Kawabata, K., K. Xu y G. Carrin (2002): Preventing impoverishment through protection against catastrophic health expenditure, Boletín de la Organización Mundial de la Salud, vol. 80, $\mathrm{N}^{\mathrm{o}} 8$, Ginebra, Organización Mundial de la Salud.

Kilsztajn, S., M.B. Camara y M.S.N. Carmo (2002): Private health expenditures and income distribution in Brazil, Revista da Associação Médica Brasileira, vol. 48, № 3, São Paulo, Associação Médica Brasileira.

Lima-Costa, M.F., S.M. Barreto y L. Giatti (2003): Health status, physical functioning, health services utilization, and expenditures on medicines among Brazilian elderly: a descriptive study using data from the National Household Survey, Cadernos de saúde pública, vol. 19, No 3, Rio de Janeiro, Escola Nacional de Saúde Pública.

Lóbrega, O.T., A.R. Marques y otros (2007): Retail prices of essential drugs in Brazil: an international comparison, Revista Panamericana de Salud Pública, vol. 22, No 2, Washington, D.C., Organización Panamericana de la Salud.

Malta, D.C., L.C.O. Cecílio y otros (2004): Perspectives of the regulation in the health insurance face the model assistance, Ciência \& saúde coletiva, vol. 9, $\mathrm{N}^{\circ} 2$, Rio de Janeiro, Associação Brasileira de Pós-Graduação em Saúde Coletiva.

Marinhol, A. y S.S. Cardoso (2007): Um estudo multinível sobre as filas para internações relacionadas com a gravidez, o parto e o puerpério no sus, Economia aplicada, vol. 11, № 4, São Paulo, Economia Aplicada.

Mendoza-Sassi, R., J.U. Béria y A.J.D. Barros (2003): Outpatient health service utilization and associated factors: a populationbased study, Revista de Saúde Pública, vol. 37, № 3, São Paulo, Facultad de Salud Pública, Universidad de São Paulo.

Ministerio de Salud de Brasil (2004): Datasus, Brasilia. Disponible en: www.datasus.gov.br.

Murray, M.P. (2006): Avoiding invalid instruments and coping with weak instruments, Journal of Economic Perspectives, vol. 20, $\mathrm{N}^{\mathrm{o}}$ 4, Nashville, Tennessee, American Economic Association.

Murray, C.J.L., K. Xu y otros (2003): Assessing the distribution of household financial contributions to the health system: concepts and empirical application, en C.J.L. Murray y D.B. Evans (comps), Health Systems Performance Assessment: Debates, Methods and Empiricism, Ginebra, Organización Mundial de la Salud.

Neri, M. y W. Soares (2002): Social inequality and health in Brazil, Cadernos de saúde pública, vol. 18, suplemento, Rio de Janeiro, Escola Nacional de Saúde Pública.

Ocké-Reis, C.O. (2005): Challenges of the regulation of Brazilian private health insurance, Public Finance and Management, vol. 5, No 4, Harrisburg, Southern Public Administration Education Foundation Inc.

Ocké-Reis, C.O. y S.S. Cardoso (2006): Uma descrição do comportamento dos preços dos planos de assitência à saúde, documento de discusión, $\mathrm{N}^{\circ} 1232$, Rio de Janeiro, Instituto de Investigación Económica Aplicada (IPEA).

Ocké-Reis, C.O., F.G. Silveira y M.F.S. Andreazzi (2002): Avaliação dos gastos das famílias com a assistência médica no Brasil: o caso dos planos de saúde, documento de discusión, $\mathrm{N}^{\circ}$ 921, Río de Janeiro, Instituto de Investigación Económica Aplicada.

oms (Organización Mundial de la Salud) (2000): Informe sobre la salud en el mundo 2000. Mejorar el desempeño de los sistemas de salud, Ginebra.

ops (Organización Panamericana de la Salud) (2003): La salud en las Américas 2002, Washington, D.C.

(2005): Brazil: Health System Profile, Brasilia.

(2007): Health in the Americas, vol. 2, Washington, D.C.

Pinheiro, R.S. y C. Travassos (1999): Inequality in health care use by the elderly in three district in the city of Rio de Janeiro, Cadernos de saúde pública, vol. 15, Nº 3, Rio de Janeiro, Escola Nacional de Saúde Pública. 
Pinto, L.F. y D.R. Soranz (2004): Private health plans: populational coverage in Brazil, Ciência \& saúde coletiva, vol. 9, № 1, Rio de Janeiro, Associação Brasileira de Pós-Graduação em Saúde Coletiva.

Porto, S.M., I.S. Santos y M.A.D. Ugá (2006): An analysis of health services utilization, by financing system, Ciência e saúde coletiva, vol. 11, $\mathrm{N}^{\circ}$ 4, Rio de Janeiro, Associação Brasileira de Pós-Graduação em Saúde Coletiva.

Ribeiro, M.C.S., R.B. Barata y otros (2006): Sociodemographic profile and utilization patterns of the public health system (SUS) - PNAD 2003, Ciência \& saúde coletiva, vol. 11, № 4, Rio de Janeiro, Associação Brasileira de Pós-Graduação em Saúde Coletiva.

Rocha, J.S.Y. y J.G. Simões (1999): Study of public and private hospital care on a populational basis, 1986-1996, Revista de Saúde Pública, vol. 33, No 1, São Paulo, Facultad de Salud Pública, Universidad de São Paulo.

Sekhri, N. y W. Savedoff (2005): Private health insurance: implications for developing countries, Boletín de la Organización Mundial de la Salud, vol. 83, N 2, Ginebra, Organización Mundial de la Salud.

(2006): Regulating private health insurance to serve the public interest: policy issues for developing countries, International Journal of Health Planning and Management, vol. 21, $\mathrm{N}^{\circ} 4$, Keele, Centre for Health Planning and Management, Keele University.

Silveira, F.G., R.G. Osório y S.F. Piola (2002): Families' expenses with health, Ciência \& saúde coletiva, vol. 7, № 4, Rio de Janeiro, Associação Brasileira de Pós-Graduação em Saúde Coletiva.
Solla, J.J.S.P., A.A.C. Reis y otros (2007): Recent changes of the Brazilian public health system federal financing: primary health, Revista brasileira de saúde materna e infantil, vol. 7, $\mathrm{N}^{\circ} 4$, Pernambuco, Instituto Materno Infantil de Pernambuco.

StataCorp. (2005): Intercooled Stata 9.0, College Station (TX).

Strauss, J. y D. Thomas (1998): Health, nutrition and economic development, Journal of Economic Literature, vol. 36, $\mathrm{N}^{\mathrm{o}} 2$, Nashville, Tennessee, American Economic Association.

Viacava, F., P.R.B. Souza-Júnior y C.L. Szwarcwald (2005): Coverage of the Brazilian population 18 years and older by private health plans: an analysis of data from the World Health Survey, $\mathrm{Ca}$ dernos de saúde pública, vol. 21, suplemento, Rio de Janeiro, Escola Nacional de Saúde Pública.

Van de Ven, W.P.M.M. y B.M.S. Van Pragg (1981): The demand for deductibles in private health insurance: a probit model with sample selection, Journal of Econometrics, vol. 17, $\mathrm{N}^{\mathrm{o}} 2$, Amsterdam, Elsevier.

Waters, H.R. (2000): Measuring equity in access to health care, Social Science \& Medicine, vol. 15, Amsterdam, Elsevier.

Waters, H.R., G.F. Anderson y J. Mays (2004): Measuring financial protection in health in the United States, Health Policy, vol. 69 , $\mathrm{N}^{\mathrm{o}}$ 3, Amsterdam, Elsevier.

Xu, K., D.B. Evans y otros (2003): Household catastrophic health expenditure: a multicountry analysis, The Lancet, vol. 362, $\mathrm{N}^{\circ}$ 9378, Amsterdam, Elsevier. 